\title{
On the Cohomology of Topological Semigroups
}

\author{
Maysam Maysami Sadr ${ }^{1 *}$, Danial Bouzarjomehri Amnieh²
}

\begin{abstract}
In this short note, we give some new results on continuous bounded cohomology groups of topological semigroups with values in complex field. We show that the second continuous bounded cohomology group of a compact metrizable semigroup, is a Banach space. Also, we study cohomology groups of amenable topological semigroups, and we show that cohomology groups of rank greater than one of a compact left or right amenable semigroup, are trivial. Also, we give some examples and applications about topological lattices.
\end{abstract}

Keywords: Banach homology, Bounded cohomology, Topological semigroup.

2010 AMS: 20J06, 22A99, 20J05, 22A26.

${ }^{1}$ Department of Mathematics, Institute for Advanced Studies in Basic Sciences, Zanjan, Iran, sadr@iasbs.ac.ir, ORCID: 0000-0003-0747-4180

${ }^{2}$ Department of Mathematics, Institute for Advanced Studies in Basic Sciences, Zanjan, Iran, danial.bouzarj@iasbs.ac.ir, ORCID.

0000-0002-0883-6510

*Corresponding author: sadr@iasbs.ac.ir

Received: 21 January 2019, Accepted: 29 July 2019, Available online: 30 September 2019

\section{Introduction}

Homology theory is one of the most powerful tools for study of various mathematical objects. There are many kind of (co)homology theories, for instance: de Rham (co)homology for smooth manifolds, sheaf cohomology for algebraic varieties, singular (co)homology of topological spaces with values in an arbitrary ring, Čech cohomology for topological spaces, bordism homology, and Hochschild (co)homology for rings and topological algebras with values in bimodules.

The bounded cohomology theory was first defined for discrete groups by B. E. Johnson [1] and F. Trauber. Then, M. Gromov [2] extended it to topological spaces. Gromov has been proved that for every path connected manifold, the bounded cohomology group of any rank is equivalent with bounded cohomology group of fundamental group of the manifold with the same rank; for more details one can look [3]. The continuous cohomology theory for topological spaces and topological groups have been studied by many mathematicians in different approaches; see [4, 5, 6] and [7].

The bounded continuous cohomology theory for topological spaces and topological groups, generalizing both continuous cohomology and bounded cohomology theories simultaneously, has been studied by many authors such as R. Frigerio [8] and N. Monod [9]. Bounded cohomology of semigroups has also been considered by many mathematicians such as R. Brooks [10], R. I. Grigorchuk [11] and N. V. Ivanov [12].

In this paper we establish a topological bounded cohomology theory for topological semigroups, using continuous bounded cocycles.

In the section 2, we define bounded continuous cohomology group of a topological semigroup. In the section 3 , we show some basic properties of the bounded continuous cohomology. In the next section, we explain the bounded continuous cohomology relation with amenability. In the last section, we give some examples of it. 


\section{Definition of the cohomology}

For any set $X, \mathbf{B}(X)$ denotes the Banach space of all bounded complex $(\mathbb{C})$ valued maps on $X$ with the uniform norm. If $X$ has a topology, then $\mathbf{C}(X) \subset \mathbf{B}(X)$ denotes the Banach subspace of continuous maps. By a topological semigroup we mean a semigroup $S$ with a topology such that the multiplication $S \times S \rightarrow S$ is jointly continuous.

Let $S$ be a semigroup. Let $\mathscr{C}_{b}^{0}(S)=\mathbb{C}$, and for $n \geq 1$, let $\mathscr{C}_{b}^{n}(S)=\mathbf{B}\left(S^{n}\right)$. The elements of $\mathscr{C}_{b}^{n}(S)$ are called bounded cochains of the semigroup $S$. Let $\delta^{0}: \mathscr{C}_{b}^{0}(S) \rightarrow \mathscr{C}_{b}^{1}(S)$ be the zero linear map and for $n \geq 1$, define the bounded linear map $\delta^{n}: \mathscr{C}_{b}^{n}(S) \rightarrow \mathscr{C}_{b}^{n+1}(S)$ by

$$
\delta^{n}(f)\left(s_{1}, \ldots, s_{n+1}\right)=f\left(s_{2}, \ldots, s_{n+1}\right)+\sum_{i=1}^{n}(-1)^{i} f\left(s_{1}, \ldots, s_{i} s_{i+1}, \ldots, s_{n+1}\right)+(-1)^{n+1} f\left(s_{1}, \ldots, s_{n}\right),
$$

for $f \in \mathscr{C}_{b}^{n}(S)$ and $s_{1}, \ldots, s_{n+1} \in S$. The linear map $\delta^{n}$ is called coboundary. It is easily checked that $\delta^{n+1} \delta^{n}=0$ and thus, we have the following cochain complex of Banach spaces and bounded linear maps:

$$
0 \longrightarrow \mathscr{C}_{b}^{0}(S) \stackrel{\delta^{0}}{\longrightarrow} \mathscr{C}_{b}^{1}(S) \stackrel{\delta^{1}}{\longrightarrow} \cdots \stackrel{\delta^{n-1}}{\longrightarrow} \mathscr{C}_{b}^{n}(S) \stackrel{\delta^{n}}{\longrightarrow} \cdots
$$

Then, the cohomology groups of the complex (2.1) are called bounded cohomology groups of $S$ and denoted by $\mathscr{H}_{b}^{n}(S)=\frac{\operatorname{ker} \delta^{n}}{\operatorname{Im} \delta^{n-1}}$. Always, the quotient vector space $\mathscr{H}_{c b}^{n}(S)$ is considered as a semi normed space with quotient semi norm. As any cohomology theory, we let $\mathscr{B}_{b}^{n}(S)=\operatorname{Im} \delta^{n-1}$ and $\mathscr{Z}_{b}^{n}(S)=\operatorname{ker} \delta^{n}$. The elements of $\mathscr{B}_{b}^{n}$ and $\mathscr{Z}_{b}^{n}$ are called bounded $n$-coboundaries and bounded $n$-cocycles, respectively. For more details on bounded cohomology of semigroups, see [11].

Now, suppose that $S$ is a topological semigroup. Let $\mathscr{C}_{c b}^{0}(S)=\mathbb{C}$, and for $n \geq 1$, let $\mathscr{C}_{c b}^{n}(S)=\mathbf{C}\left(S^{n}\right)$. The elements of $\mathscr{C}_{c b}^{n}(S)$ are called continuous bounded cochains of the semigroup $S$. Then we have the following Banach subcomplex of (2.1):

$$
0 \longrightarrow \mathscr{C}_{c b}^{0}(S) \stackrel{\delta^{0}}{\longrightarrow} \mathscr{C}_{c b}^{1}(S) \stackrel{\delta^{1}}{\longrightarrow} \cdots \stackrel{\delta^{n-1}}{\longrightarrow} \mathscr{C}_{c b}^{n}(S) \stackrel{\delta^{n}}{\longrightarrow} \cdots
$$

Definition 2.1. The cohomology groups of the complex (2.2) are called continuous bounded cohomology groups of $S$ and denoted by $\mathscr{H}_{c b}^{n}(S)$.

Analogously, we have the space of continuous bounded $n$-coboundaries $\mathscr{B}_{c b}^{n}(S)$, and the space of continuous bounded $n$-cocycles $\mathscr{Z}_{c b}^{n}$, and $\mathscr{H}_{c b}^{n}(S)$ is considered by the quotient semi norm.

Remark 2.2. (I) Let $S$ be a discrete semigroup. Consider the convolution Banach algebra $\ell^{1}(S)$. Then the space $\mathbb{C}$ is a Banach $\ell^{1}(S)$-bimodule by the symmetric action $f \cdot \lambda=\lambda \cdot f=\lambda \sum_{s \in S} f(s)$ for $f \in \ell^{1}(S)$ and $\lambda \in \mathbb{C}$. It is well known and easily checked that the bounded Hochschild cohomology groups of $\ell^{1}(S)$ with values in the bimodule $\mathbb{C}$ and the bounded cohomology groups of $S$ are isometric isomorph. Thus, the bounded cohomology is a special case of Hochschild cohomology, see[13].

(II) Let $S$ be a compact Hausdorff semigroup. If we dualize cochain complex (2.2), then (by the natural isomorphism between $\mathbf{C}(X)^{*}$ and the Banach space of complex Borel regular measures $\mathbf{M}(X)$ for any compact Hausdorff space $\left.X\right)$ we have the chain complex

$$
0<\quad \mathbb{C} \ll\left(^{\left(\delta^{0}\right)^{*}} \mathbf{M}(S) \stackrel{\left(\delta^{1}\right)^{*}}{\longleftarrow} \mathbf{M}\left(S^{2}\right) \stackrel{\left(\delta^{2}\right)^{*}}{\longleftarrow} \cdots\right.
$$

One can consider the homology of this complex as a measure homology theory $(c f .[14,15])$ that is a topological version of $\ell^{1}$-homology of discrete semigroups [11].

\section{Some basic properties}

Theorem 3.1. Let $S, T$ be topological semigroups and $\phi: S \rightarrow T$ be a continuous homomorphism. Then for every $n \geq 0$, there is a canonical continuous linear map

$$
\mathscr{H}_{c b}^{n}(\phi): \mathscr{H}_{c b}^{n}(T) \rightarrow \mathscr{H}_{c b}^{n}(S)
$$


Proof. For every $n \geq 1$ let $\hat{\phi}_{n}: \mathscr{C}_{c b}^{n}(T) \rightarrow \mathscr{C}_{c b}^{n}(S)$ be defined by

$$
\hat{\phi}_{n}(f)\left(s_{1}, \ldots, s_{n}\right)=f\left(\phi\left(s_{1}\right), \ldots, \phi\left(s_{n}\right)\right) \quad\left(f \in \mathscr{C}_{c b}^{n}(T)\right) .
$$

Then $\left(\hat{\phi}_{n}\right)_{n}$ is a cochain map between continuous bounded cohomology complexes of $T$ and $S$, i.e. the following diagram is commutative:



Thus, the standard arguments of Banach homology ([16], [17]) shows that we have a continuous linear map $\mathscr{H}_{c b}^{n}(\phi)$, defined by

$$
\mathscr{H}_{c b}^{n}(\phi)\left(f+\mathscr{B}_{c b}^{n}(T)\right)=\hat{\phi}_{n}(f)+\mathscr{B}_{c b}^{n}(S),
$$

for $f \in \mathscr{Z}_{c b}^{n}(T)$.

Let $\mathscr{T} \mathscr{S} \mathscr{G}$ be the category of topological semigroups and continuous homomorphisms. Then, the above theorem shows that $\mathscr{H}_{c b}^{n}$ is a contravariant functor from $\mathscr{T} \mathscr{S} \mathscr{G}$ to the category of seminormed spaces and continuous linear maps. Since the category $\mathscr{T} \mathscr{S} \mathscr{G}$ has no additive properties, the computation of continuous bounded cohomology groups often are very hard. In another paper, we will consider various extensions of $\mathscr{H}_{c b}^{n}$ to some categories of representations of topological semigroups on topological vector spaces.

For any topological semigroup $S$ it is trivial that $\mathscr{H}_{c b}^{0}(S)=\mathbb{C}$. First order cohomology groups are zero:

Theorem 3.2. For any topological semigroup $S, \mathscr{H}_{c b}^{1}(S)$ is zero.

Proof. Let $f \in \mathscr{Z}_{c b}^{1}(S)$ be a 1-cocycle. Then for every $s, t \in S$, we have $\delta^{1}(f)(s, t)=f(t)-f(s t)+f(s)=0$ and thus,

$$
f(s t)=f(s)+f(t) .
$$

In particular, for every $s \in S$ and $n \in \mathbb{N}$, we have $f\left(s^{n}\right)=n f(s)$. This implies that $f(s)=0$, since $f$ is a bounded map. Therefore $\mathscr{Z}_{c b}^{1}(S)=0$ and $\mathscr{H}_{c b}^{1}(S)$ is zero.

We recall a kind of limiting process: Let $E$ be the Banach space of all bounded sequences of complex numbers with uniform norm and let $F \subset E$ be the subspace of all convergent sequences. Then, the functional $\lim : F \rightarrow \mathbb{C}$ defined by $\lim \left(a_{n}\right)_{n \in \mathbb{N}}=\lim _{n \rightarrow \infty} a_{n}$ is a bounded functional and thus, by the Hann-Banach theorem there is a bounded functional LIM : $E \rightarrow \mathbb{C}$ that extends lim and $\|$ LIM $\|=1$ (such functionals are called Banach limits).

Theorem 3.3. Let $S$ be a compact semigroup with a metric $d$ that induces the topology of $S$ and has the following property:

- For every $\beta>0, s, t \in S$, and $i \in \mathbb{N}$ if $d(s, t)<\beta$ then $d\left(s^{i}, t^{i}\right)<\beta$.

Then $\mathscr{H}_{c b}^{2}(S)$ is a Banach space.

Proof. It is enough that we prove $\delta^{1}\left(\mathscr{C}_{c b}^{1}(S)\right)$ is closed in $\mathscr{C}_{c b}^{2}(S)$, and thus, it is sufficient to construct a bounded linear map $\gamma: \mathscr{C}_{c b}^{2}(S) \rightarrow \mathscr{C}_{c b}^{1}(S)$ such that $\gamma \delta=i d_{\mathscr{C}_{c b}^{1}}(S)$.

Let $f \in \mathscr{C}_{c b}^{2}(S)$ be a 2-cochain. For every $s \in S$, consider the bounded sequence $a_{n}^{f, s}=n^{-1} \sum_{i=1}^{n-1} f\left(s^{i}, s\right)(n \geq 2)$ of complex numbers and define $\gamma(f)(s)=\operatorname{LIM}\left(a_{n}^{f, s}\right)$. Let $\alpha>0$ be arbitrary. Since $S^{2}$ is a compact metric space and $f$ is continuous, there is $\beta>0$ such that if $d\left(t_{1}, t_{2}\right)<\beta$ and $d\left(t_{1}^{\prime}, t_{2}^{\prime}\right)<\beta$ then $\left|f\left(t_{1}, t_{1}^{\prime}\right)-f\left(t_{2}, t_{2}^{\prime}\right)\right|<\alpha$. This property together with (*) implies that for every $s, t \in S$ and $n \in \mathbb{N}$ if $d(s, t)<\beta$ then $\left|a_{n}^{f, s}-a_{n}^{f, t}\right|<\alpha$ and thus, $|\gamma(f)(s)-\gamma(f)(t)|<\alpha$. Therefore we have proved $\gamma(f)$ is continuous and $\gamma(f) \in \mathscr{C}_{c b}^{1}(S)$. Also, it is easily checked that $\gamma$ is a bounded linear operator.

Now, suppose that $g$ is in $\mathscr{C}_{c b}^{1}(S)$. For every $s \in S$ and $i \geq 1$ we have

$$
\delta^{1}(g)\left(s^{i}, s\right)=g\left(s^{i}\right)-g\left(s^{i+1}\right)+g(s),
$$

thus, for every $n \geq 2, a_{n}^{\delta^{1}(g), s}=g(s)-n^{-1} g\left(s^{n}\right)$. Therefore we have

$$
\gamma\left(\delta^{1}(g)\right)(s)=\operatorname{LIM}\left(a_{n}^{\delta^{1}}(g), s\right)=\lim _{n \rightarrow \infty} g(s)-n^{-1} g\left(s^{n}\right)=g(s) .
$$

Thus, we have proved $\gamma \delta(g)=g$. 
It is easily checked that the arguments of the proof of Theorem 3.3, satisfy when $S$ is a discrete semigroup:

Theorem 3.4. Let $S$ be a discrete semigroup. Then $\mathscr{H}_{b}^{2}(S)$ is a Banach space.

Proposition 3.5. Let $S$ and $T$ be topological semigroups and $p: S \times T \rightarrow S$ be the natural projection. Suppose that $T$ has a unite element $e$. Then the linear map $\mathscr{H}_{c b}^{n}(p): \mathscr{H}_{c b}^{n}(S) \rightarrow \mathscr{H}_{c b}^{n}(S \times T)$ is injective for all $n \geq 1$.

Proof. For $n=1$, the result follows from Theorem 3.2. Let $n \geq 2$ be fixed, and let $p^{(n)}:(S \times T)^{n} \rightarrow S^{n}$ be defined by

$$
p^{(n)}\left(\left(s_{1}, t_{1}\right), \ldots,\left(s_{n}, t_{n}\right)\right)=\left(s_{1}, \ldots, s_{n}\right)
$$

for $s_{1}, \ldots, s_{n} \in S, t_{1}, \ldots, t_{n} \in T$. By definition of $\mathscr{H}_{c b}^{n}(p)$, we must prove that if $f \in \mathscr{Z}_{c b}^{n}(S)$ and $f \circ p^{(n)} \in \mathscr{B}_{c b}^{n}(S \times T)$, then $f$ is in $\mathscr{B}_{c b}^{n}(S)$. Thus, consider such a $n$-cocycle $f$. There is $g \in \mathscr{C}_{c b}^{n-1}(S \times T)$ such that $\delta^{n-1}(g)=f \circ p^{(n)}$. Define $\hat{g} \in \mathscr{C}_{c b}^{n-1}(S)$ by

$$
\hat{g}\left(s_{1}, \ldots, s_{n-1}\right)=g\left(\left(s_{1}, e\right), \ldots,\left(s_{n-1}, e\right)\right) \quad\left(s_{1}, \ldots, s_{n-1} \in S\right) .
$$

Then, for every $s_{1}, \ldots, s_{n} \in S$, we have

$$
\begin{aligned}
\delta^{n-1}(\hat{g})\left(s_{1}, \ldots, s_{n}\right) & =\hat{g}\left(s_{2}, \ldots, s_{n}\right)+\sum_{i=1}^{n-1}(-1)^{i} \hat{g}\left(s_{1}, \ldots, s_{i} s_{i+1}, \ldots, s_{n}\right)+(-1)^{n} \hat{g}\left(s_{1}, \ldots, s_{n-1}\right)=g\left(\left(s_{2}, e\right), \ldots,\left(s_{n}, e\right)\right) \\
& +\sum_{i=1}^{n-1}(-1)^{i} g\left(\left(s_{1}, e\right), \ldots,\left(s_{i} s_{i+1}, e\right), \ldots,\left(s_{n}, e\right)\right)+(-1)^{n} g\left(\left(s_{1}, e\right), \ldots,\left(s_{n-1}, e\right)\right) \\
& =\delta^{n-1}(g)\left(\left(s_{1}, e\right), \cdots,\left(s_{n}, e\right)\right) .
\end{aligned}
$$

On the other hand, $\delta^{n-1}(g)\left(\left(s_{1}, e\right) \ldots,\left(s_{n}, e\right)\right)=f\left(s_{1}, \ldots, s_{n}\right)$. Thus, we have $\delta^{n-1}(\hat{g})=f$ and $f \in \mathscr{B}_{c b}^{n}(S)$.

\section{Relation with amenability}

Let $S$ be a topological semigroup. A function $f \in \mathbf{C}(S)$ is called right uniformly continuous, if the map $\Phi_{f}: S \rightarrow \mathbf{C}(S)$ defined by $\Phi_{f}(s)=f \cdot s$ is continuous with uniform norm of $\mathbf{C}(S)$, where $f \cdot s(x)=f(s x)(x \in S)$. Left uniformly continuous functions are similarly defined. The space of all right (left) uniformly continuous functions is denoted by $\mathbf{R U C}(S)(\mathbf{L U C}(S))$. Note that if $f \in \mathbf{R U C}(S)$ and $s \in S$, then $f \cdot s \in \mathbf{R U C}(S)$. Also, it is easily checked that $\mathbf{R U C}(S)=\mathbf{L U C}(S)=\mathbf{C}(S)$ when $S$ is compact, and it is clear that $\mathbf{R U C}(S)=\mathbf{L U C}(S)=\mathbf{C}(S)=\mathbf{B}(S)$ when $S$ is discrete.

A topological semigroup $S$ is called it left amenable if there is a left invariant mean on $\mathbf{R U C}(S)$, i.e. a bounded linear functional $m$ on $\mathbf{R U C}(S)$ such that $\left\langle m, 1_{S}\right\rangle=\|m\|=1$ (where $1_{S}$ is the constant map on $S$ with value 1 ) and for every $s \in S$ and $f \in \mathbf{R U C}(S),\langle m, f \cdot s\rangle=\langle m, f\rangle$. Right invariant means and right amenable semigroups are similarly defined. A topological semigroup is called amenable if it is both left and right amenable.

It is well known and easily checked that for topological semigroups $S$ and $T$, if there is a continuous homomorphisms form $S$ onto $T$, and $S$ is left (right) amenable, then $T$ is also left (right) amenable. In particular, if $S$ is left (right) amenable semigroup with topology $\tau$, and $\tau^{\prime}$ is another semigroup topology on $S$ such that $\tau^{\prime} \subset \tau$, then $\left(S, \tau^{\prime}\right)$ is left (right) amenable. Thus, any commutative topological semigroup is amenable since any commutative discrete semigroup is amenable ([18]). It is well known that any compact group is amenable ([18]), but there are compact semigroups that are not left amenable nor right amenable:

Example 4.1. Let $X$ and $Y$ be two disjoint compact spaces with distinguished elements $x_{0} \in X$ and $y_{0} \in Y$. Define a semigroup multiplication on disjoint union space $T=X \cup Y$ by

$$
x x^{\prime}=x_{0}, \quad y y^{\prime}=y_{0}, \quad x y=x_{0}, \quad y x=y_{0},
$$

for every $x, x^{\prime} \in X$ and $y, y^{\prime} \in Y$. Then $T$ becomes a compact semigroup. We show that $T$ is not left amenable. Suppose $m$ is a bounded linear functional on $\mathbf{C}(T)$ such that $\left\langle m, 1_{T}\right\rangle=\|m\|=1$. For every $x \in X \subset T$ and $f \in \mathbf{C}(T)$, we have $\langle m, f \cdot x\rangle=\left\langle m, f\left(x_{0}\right) 1_{T}\right\rangle=f\left(x_{0}\right)$, and similarly $\langle m, f \cdot y\rangle=f\left(y_{0}\right)$ for every $y \in Y$. Thus, $m$ is not a left invariant mean, since there is a continuous map $f$ on $S$ such that $f\left(x_{0}\right) \neq f\left(y_{0}\right)$. Thus, we have proved that $T$ is not left amenable. Let $T^{o p}$ be the opposite semigroup of $T$. Then $T^{o p}$ is not right amenable. Now the compact semigroup $S=T \times T^{o p}$ is not left nor right amenable, since the canonical projection maps from $S$ to $T$ and $T^{o p}$ are continuous surjective homomorphisms.

We need the following simple topological lemma. 
Lemma 4.2. Let $X$ be a topological space and $Y$ be a compact space. Let $f: X \times Y \rightarrow \mathbb{C}$ be a continuous map. Then $F: X \rightarrow \mathbf{C}(Y)$, defined by $F(x)(y)=f(x, y)$ is continuous with norm topology of $\mathbf{C}(Y)$.

Proof. Let $x_{0} \in X$ and $\alpha>0$ be arbitrary. Since $f$ is continuous, for every $y \in Y$, there are open sets $U_{y}, V_{y}$ in $X$ and $Y$ respectively, such that $\left(x_{0}, y\right) \in U_{y} \times V_{y}$ and $\left|f\left(x_{0}, y\right)-f\left(x, y^{\prime}\right)\right|<\alpha / 2$ for every $\left(x, y^{\prime}\right) \in U_{y} \times V_{y}$. Since $Y$ is compact, there are $y_{1}, \ldots, y_{n} \in Y$ such that $Y=\cup_{i=1}^{n} V_{y_{i}}$. Let $W$ be the open set $\cap_{i=1}^{n} U_{y_{i}}$. Let $x \in W$ and $y \in Y$ be arbitrary. Then for some $i$ $(i=1, \cdots, n), y$ belongs to $V_{y_{i}}$ and we have,

$$
\left|f(x, y)-f\left(x_{0}, y\right)\right| \leq\left|f(x, y)-f\left(x_{0}, y_{i}\right)\right|+\left|f\left(x_{0}, y_{i}\right)-f\left(x_{0}, y\right)\right|<\alpha / 2+\alpha / 2=\alpha .
$$

Thus, we have $\left\|F(x)-F\left(x_{0}\right)\right\|<\alpha$ for every $x \in W$. The proof is complete.

The proof of the following Theorem is an adaptation of the proof given in [11, Theorem 2.1] to the topological case.

Theorem 4.3. Let $S$ be a compact semigroup and suppose that $S$ is left (right) amenable. Then $\mathscr{H}_{c b}^{n}$ is zero for every $n \geq 0$.

Proof. Suppose that $S$ is left amenable and let $m$ be a left invariant mean on $\mathbf{C}(S)^{*}$. Similar [11], we use the notation

$$
m(f)=\int_{S} f(s) d(s)
$$

for $f \in \mathbf{C}(S)$. Thus, we have

(i) $\int_{S} 1_{S}(s) d(s)=1$, and

(ii) $\int_{S} f(t s) d(s)=\int_{S} f(s) d(s)$ for every $f \in \mathbf{C}(S)$ and $t \in S$.

The cases $n=0$ and $n=1$ were considered before, thus, suppose that $n \geq 2$ and let $f \in \mathscr{Z}_{c b}^{n}(S)$. Then, for every $s_{1}, \ldots, s_{n+1} \in S$, we have,

$$
\delta^{n}(f)\left(s_{1}, \ldots, s_{n+1}\right)=f\left(s_{2}, \ldots, s_{n+1}\right)+\sum_{i=1}^{n}(-1)^{i} f\left(s_{1}, \ldots, s_{i} s_{i+1}, \ldots, s_{n+1}\right)+(-1)^{n+1} f\left(s_{1}, \ldots, s_{n}\right)=0
$$

If we fix $s_{1}, \ldots, s_{n} \in S$ and integrate the above formula over the variable $s_{n+1}$ in the sense of (4.1), then we have

$$
\begin{aligned}
\int_{S} f\left(s_{2}, \ldots, s_{n+1}\right) d\left(s_{n+1}\right) & +\sum_{i=1}^{n-1}(-1)^{i} \int_{S} f\left(s_{1}, \ldots, s_{i} s_{i+1}, \ldots, s_{n}, s_{n+1}\right) d\left(s_{n+1}\right) \\
& +(-1)^{n} \int_{S} f\left(s_{1}, \ldots, s_{n-1}, s_{n} s_{n+1}\right) d\left(s_{n+1}\right)+(-1)^{n+1} \int_{S} f\left(s_{1}, \cdots, s_{n}\right) d\left(s_{n+1}\right)=0
\end{aligned}
$$

By property (i),

$$
\int_{S} f\left(s_{1}, \ldots, s_{n}\right) d\left(s_{n+1}\right)=f\left(s_{1}, \ldots, s_{n}\right),
$$

and by property (ii),

$$
\int_{S} f\left(s_{1}, \ldots, s_{n-1}, s_{n} s_{n+1}\right) d\left(s_{n+1}\right)=\int_{S} f\left(s_{1}, \ldots, s_{n-1}, s_{n+1}\right) d\left(s_{n+1}\right) .
$$

Let $g: S^{n-1} \rightarrow \mathbb{C}$ be defined by

$$
g\left(s_{2}, \ldots, s_{n}\right)=\int_{S} f\left(s_{2}, \ldots, s_{n}, s_{n+1}\right) d\left(s_{n+1}\right) .
$$

By Lemma 4.2, the map $F: S^{n-1} \rightarrow \mathbf{C}(S)$, defined by

$$
F\left(s_{2}, \ldots, s_{n}\right)(x)=f\left(s_{2}, \ldots, s_{n}, x\right) \quad(x \in S),
$$

is continuous with the norm of $\mathbf{C}(S)$. On the other hand, $\int: \mathbf{C}(S) \rightarrow \mathbb{C}$ is also continuous with the norm. Thus, the map $g=\int F$ is in $\mathscr{C}_{c b}^{n-1}(S)$. Therefore, by (4.2), (4.3) and (4.4), we have,

$$
(-1)^{n} f\left(s_{1}, \ldots, s_{n}\right)=g\left(s_{2}, \ldots, s_{n}\right)+\sum_{i=1}^{n-1}(-1)^{i} g\left(s_{1}, \ldots, s_{i} s_{i+1}, \ldots, s_{n}\right)+(-1)^{n} g\left(s_{1}, \ldots, s_{n-1}\right)
$$

But the right hand side of the latter equation is $\delta^{n-1}(g)$. Thus,

$$
f=\delta^{n-1}\left((-1)^{n} g\right) .
$$

Therefore we have proved $\mathscr{B}_{c b}^{n}(S)=\mathscr{Z}_{c b}^{n}(S)$ and $\mathscr{H}_{c b}^{n}(S)=0$. A similar proof can be given in the case of right amenable $S$. 


\section{Some examples}

Gromov ([2]) proved that for any connected manifold $X$, and any $n \geq 1$, the bounded cohomology of $X$ and the bounded cohomology of the fundamental homotopy group $\pi_{1}(X)$ of $X$ coincide (for more details see [2,10,12], and [11]). Thus, there are many discrete groups that their bounded cohomology groups are non zero.

Let $G$ be a discrete group and $S$ be a topological semigroup with a unite. Suppose that for an integer $n \geq 2, \mathscr{H}_{c b}^{n}(G) \neq 0$ (for example $G=F_{2}$, the free group on two generators, and $n=2$, see [11], [19]). Then by Proposition 3.5 we have $\mathscr{H}_{c b}^{n}(G \times S) \neq 0$.

A semigroup $S$ is called semilattice if it is commutative and $s s=s$ for every $s \in S$.

Theorem 5.1. Let $S$ be a topological semilattice. Then $\mathscr{H}_{c b}^{2}(S)$ is zero.

Proof. Let $f \in \mathscr{Z}_{c b}^{2}(S)$ be a 2 -cocycle. We need a $g \in \mathscr{C}_{c b}^{1}(S)$ such that for every $s, t \in S$,

$$
f(s, t)=g(s)+g(t)-g(s, t) .
$$

Since $f$ is a 2-cocycle, for every $s_{1}, s_{2}, s_{3} \in S$ we have

$$
\delta^{2}(f)\left(s_{1}, s_{2}, s_{3}\right)=f\left(s_{2}, s_{3}\right)-f\left(s_{1} s_{2}, s_{3}\right)+f\left(s_{1}, s_{2} s_{3}\right)-f\left(s_{1}, s_{2}\right)=0 .
$$

By applying (5.1) with $s_{1}=s, s_{2}=s, s_{3}=t$, we obtain

$$
f(s, s)=f(s, s t)
$$

and similarly

$$
f(t, t)=f(t, s t) .
$$

By applying (5.1) with $s_{1}=s, s_{2}=t, s_{3}=s t$, we obtain

$$
f(s, t)=f(t, s t)-f(s t, s t)+f(s, s t)
$$

Now, by (5.2), (5.3) and (5.4), we have

$$
f(s, t)=f(t, t)+f(s, s)-f(s t, s t) .
$$

Thus, if we define $g(s)=f(s, s)(s \in S)$, then $g \in \mathscr{C}_{c b}^{1}(S)$ and $\delta^{1}(g)=f$.

Remark 5.2. (a) The above result follows directly from Theorem 4.3, when $S$ is compact.

(b) In [20], Y. Choi proved that for any discrete semilattice S and any symmetric Banach $\ell^{1}(S)$ bimodule E, every Hochschild cohomology group of $\ell^{1}(S)$ with coefficient in $E$ is trivial (see Remark of Section 1). Thus, for every discrete semilattice $S$ and $n \geq 0, \mathscr{H}_{b}^{n}(S)=0$.

Example 5.3. Let $(X, d)$ be a metric space and let for every $A \subset X$ and $\varepsilon>0, N_{\varepsilon}(A)$ be the $\varepsilon$-neighborhood of $A$ in $X$. Let $S_{X}$ be the set of all nonempty closed bounded subsets of $X$. Then $S_{X}$ is a metric space by the following metric that is called Hausdorff distance:

$$
d_{H}\left(C_{1}, C_{2}\right)=\inf \left\{\varepsilon>0: C_{1} \subset N_{\varepsilon}\left(C_{2}\right) \text { and } C_{2} \subset N_{\varepsilon}\left(C_{1}\right)\right\},
$$

for $\left(C_{1}, C_{2} \in S_{X}\right)$. Also $S_{X}$ is a semilattice with the multiplicaton $C_{1} C_{2}=C_{1} \cup C_{2}$. It is easily checked that $S_{X}$ is a topological semilattice with the topology induced by $d_{H}$. Note that if $(X, d)$ is a compact metric space then so is $\left(S_{X}, d_{H}\right),[21$, Lemma 5.31].

Let $(X, d)$ and $(Y, \rho)$ be disjoint compact metric spaces with distinguished elements $x_{0} \in X$ and $y_{0} \in Y$. Let $S$ and $T$ be compact semigroups defined in Example of Section 3, using $X$ and $Y$. Define the metric $d^{\prime}$ on $T=X \cup Y$ by $\left.d^{\prime}\right|_{X \times X}=d,\left.d^{\prime}\right|_{Y \times Y}=\rho$ and $d^{\prime}(x, y)=1$ for $x \in X, y \in Y$. Then $T$ together with $d^{\prime}$, satisfy the conditions of Theorem 3.3. Also, S together with the maximum metric satisfy the conditions of Theorem 3.3, but it is not left nor right amenable, and thus, we can not apply Theorem 4.3, to conclude that the cohomology groups of $S$ are zero. 


\section{References}

[1] B. E. Johnson, Cohomology in Banach algebras, Mem. Amer. Math. Soc., 127 (1972).

[2] M. Gromov, Volume and bounded cohomology, Publ. Math. IHES, 56 (1982), 5-100.

[3] R. Frigerio, Bounded cohomology of discrete groups, Amer. Math. Soc., 227 (2017).

[4] E. H. Brown, R. H. Szczarba, Continuous cohomology and real homotopy type, Trans. Amer. Math. Soc., 311(1) (1989), 57-106.

[5] L. Mdzinarishvili, Continuous singular cohomology, Georgian Math. J., 16 (2009), 321-342.

[6] M. A. Mostow, Continuous cohomology of spaces with two topologies, Mem. Amer. Math. Soc., 7 (1976), 1-142.

[7] J. D. Stasheff, Continuous cohomology of groups and classifying spaces, Bull. Amer. Math. Soc., 84(4) (1978), 513-530

[8] R. Frigerio, (Bounded) Continuous cohomology and Gromov's proportionality principle, Manuscripta Math., 134(3-4) (2011), 435-474.

[9] N. Monod, Continuous bounded cohomology of locally compact groups, Lecture notes in Mathematics, no. 1758, SpringerVerlag, Berlin, 2001.

[10] R. Brooks, Some remarks on bounded cohomology, Ann. Math. Studies, 97 (1980), 53-63.

[11] R. I. Grigorchuk, Some results on bounded cohomology, Combinatorial and geometric group theory, Edinburgh, (1993), 111-163.

[12] N. V. Ivanov, Foundation of the theory of bounded cohomology, J. Soviet Math., 143 (1985), 69-109.

[13] M. M. Sadr, A. Pourabbas, Johnson amenability for topological semigroups, Iran. J. Sci. Technol. Trans. A Sci., 32(A2), (2010), 151-160.

[14] C. Löh, The Proportionality principle of simplicial volume, Diploma thesis, Universität Münster, 2004.

[15] C. Löh, Measure homology and singular homology are isometrically isomorphic, Math. Z., 253 (2006), 197-218.

[16] A. Y. Helemskii, The homology of Banach and topological algebras, Kluwer, Dordrecht, 1989.

[17] V. Runde, Lectures on amenability, Lecture Notes in Mathematics, Springer-Verlag, Berlin, 2002.

[18] A. L. T. Paterson, Amenability, Amer. Math. Soc. Providence, 1978.

[19] V. A. Faiziev, Pseudocharacters on a free group and some group constructions, Russian Math. Surveys, 43 (1988), $225-226$.

[20] Y. Choi, Cohomology of commutative Banach algebras and $\ell$-semigroup algebras, Ph.D. thesis, University of Newcastle upon Tyne, 2006.

[21] M. R. Bridson, A. Haefliger, Metric spaces of non positive curvature, Springer-Verlag, 1999. 